\title{
An Angle Estimation to Landmarks for Autonomous Satellite Navigation
}

\author{
Qing XUE ${ }^{a}$, Hongwen YANG, Jian WANG \\ ATR Key Lab, College of Electronic Science and Engineering, National University of Defense \\ Technology, Changsha, 410073, China \\ aemail: xueqing_wangyi@yeah.net
}

\begin{abstract}
Keywords: Image Matching; Satellite Position Determination; Vision Based Navigation; Angle Estimation
\end{abstract}

\begin{abstract}
Celestial navigation is a completely autonomous navigation method, but the measure based on star sensor and infrared horizon sensor has a low precision. Especially its direction to the earth measured by infrared sensor leads to great deviation. Aimed by this consideration, we propose a vision-based method to obtain the direction to the earth in this paper. In particular, direction to the center of the earth is replaced by the direction of the landmark on the ground. And the location of landmark is determined by registering single image frames to reference image with a reliable matching method. Image matching based on feature has good performance on resistance of scale and rotation variation. Experiment results verify that the autonomous navigation for satellite with camera sensor has a higher accuracy than traditional methods. Comparing with SURF (Speeded-up Robust Feature), SIFT (Scale Invariant Feature Transform) achieves a higher precision despite of a moderate loss of efficiency.
\end{abstract}

\section{Introduction}

Currently, most of the satellite orbit measurement is based on ground station, such as USB (Unified S Band) and VLBI (Very Long Baseline Interferometry) [1]. The capability of autonomous navigation is in great demand for eliminating restriction of observation arcs. So many jobs have been done on satellite autonomous navigation technology. They mainly includes the following four kinds of typical methods: the celestial navigation, the magnetic navigation, star navigation and the relative navigation between stars. Compared with others, celestial navigation is a completely autonomous navigation method [2].

The typical celestial navigation is based on star sensor and infrared horizon sensor. Its schematic diagram is shown in Figure 1. Observing the angle $\gamma$ between the direction of navigation star and the geocentric direction, orbit can be determined by the geometric relationships among the satellite, navigation star and the earth combined with the satellite orbit dynamics equation and kalman filtering methods [3]. Although this kind of navigation method which sensing horizon directly is simply and reliable, the navigation precision is lower. For the edge of the earth is not smooth, geocentric direction measured by infrared sensor leads to great deviation. Improvement on measure the direction to earth is in need. 


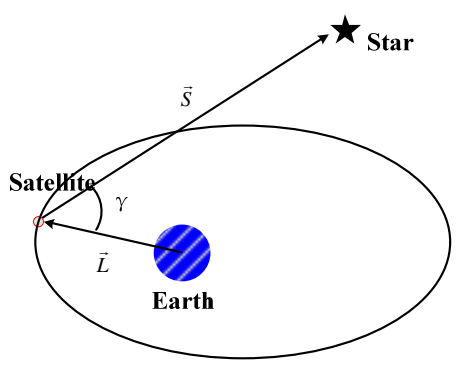

Figure 1 schematic diagram for celestial navigation

The concept of using vision to navigate is not new, researchers have been discussing the use of vision in the feedback control loop to control a robot since the 1970's [4]. And the theories developed in this field have been successfully implemented in robots for many years. Precision guidance of missile based on vision drives to maturity stage currently [5]. UAV (Unmanned aerial vehicle) is also making use of vision navigation [6]. However, this concept has by no means been completely explored. Therefore, there is little theory to describe the use of vision in space. Bevilacqua [7] computed the attitude by recovering the geometric relation between couple of subsequent frames based on an earth image registration approach. This method has high accuracy, but it can only solve relative attitude changes. Li registered satellite image with a reference image and then estimated the satellite's position according to photogrammetry [8]. This method obtain the position directly, but it can't have a high precision due to sensitivity to the height.

So we proposed a new method combining traditional celestial navigation with vision navigation. We use camera sensor instead of infrared sensor to measure the direction to the earth. Based on scene matching and photogrammetry, we can obtain a direction to the landmark on the ground. And the location of landmark is determined by registering real image to reference image with a reliable matching method based on feature, such as SIFT and SURF. Using the angle between the direction to landmark and to the star, we can determine the position of satellite by the same method in celestial navigation with the satellite orbit dynamics equation and kalman filter.

We describe the method to obtain the angle in sec.2. Image matching methods are shown in sec3. Sec. 4 present experimental results and illustration. Conclusions are given in Sect.4.

\section{Image-Based Localization Algorithm}

Navigation based on scene matching and star sensor has the observed angle between the direction of the landmark and the direction navigation star. The direction of navigation star is obtained by star sensor which is not taken consideration in this article. The direction vector to landmark on the ground is measured by scene matching method. The observed angle expose as (1):

$$
\gamma=\arccos \left(-\frac{\vec{S} \cdot \vec{L}}{L}\right)
$$

Where $\vec{S}$ is the direction to the star and $\vec{L}$ is the direction of the landmark. The main point we present in this article is the way to get the vector $\vec{L}$.

The direction of the optical center in satellite's coordinates is given value. We use the direction of the optical center as the direction vector to the landmark, and use the location of the optical center in geographic coordinates as a new landmark. Location of the optical center is determined by 
registering real image frames to reference image which is prepared with accurate location.

Analyzing the error of the method as shown:

$\Delta \gamma=\frac{\partial \gamma}{\partial \vec{L}} \Delta \vec{L}$

Where partial derivative is:

$$
\frac{\partial \gamma}{\partial \vec{L}}=\frac{\vec{S}^{T}}{\sqrt{L^{2}-\left(\vec{L}^{T} \vec{S}\right)}}\left(I_{3 \times 3}-\frac{\vec{L} \cdot \vec{L}^{T}}{L^{2}}\right)
$$

Image matching error in pixel coordinates is $\Delta z=(\Delta u, \Delta v)$, transferring into world coordinates:

$$
\Delta \vec{L}=R^{-1} Q^{-1} H\left[\begin{array}{c}
\Delta u \\
\Delta v \\
1
\end{array}\right]
$$

Where $\mathrm{Q}$ is parameter matrix of camera, $\mathrm{R}$ is rotation matrix of coordinate's transformation, and $\mathrm{H}$ is height of satellite. Image matching error is usually around 0.2 pixel. Direction vector to the star is supposed to be $(1 / \sqrt{2}, 0,1 / \sqrt{2})$, so error is $\Delta \gamma=5.69 "$. Compared with traditional celestial navigation, this method has a higher precision.

\section{Image Matching}

For the navigation based on vision, the precision of scene matching is very important. Scene matching is a typical problem of image matching, and the core process is to find the optimal geometric transformation between the two images (generally considered to be affine transformation). Common methods of image matching include matching based on gray correlation and matching based on image features. Methods based on gray correlation are greatly influenced by the imaging process, so they are not suited to multi-source image matching. In satellite navigation, real image is obtained by camera sensors on satellite, while benchmark figures are from an existing database of remote sensing images. So we choose matching method based on the image features. Features like SIFT (Scale Invariant Feature Transform) and SURF (Speeded-up Robust Feature) are widely used currently for their good performance against scaling, rotation and illumination changes.

A. Scale Invariant Feature Transform

SIFT was processed by David Lowe in 1999[9].It detects features in scale space, and use neighborhood gradient of the Key points as the main direction of the points in order to realize the independence on the scale and direction. In a two-dimensional image plane space and DOG (Difference of Gaussian) while detecting the local extrema in scale space as feature points, so that the features of uniqueness and stability of the good. Following are the major stages of computation used to match image with SIFT features:

a) Scale-space extrema detection. Using a set of continuous Gaussian convolution kernels to generate a DOG image. Comparing points in DOG scale space with its 26 neighborhood one by one, getting the local extrema and dimension as the location and scale of key points. Removing points at the edges and low contrast points to improve the matching stability and the ability to resist noise;

b) Key points localization and Orientation assignment. Using the distribution characteristics of gradient direction for neighborhood pixels to describe direction of each candidate points, a detailed model is fit to determine location and scale. Key points are selected based on measures of their 
stability. Key points for each of the specified direction makes the operator possess rotation invariance;

c) Key point descriptor. The local image gradients are measured at the selected scale in the region around each key point. These are transformed into a representation that allows for significant levels of local shape distortion and change in illumination. Each point uses $4 \times 4$ seed points to describe, finally forms the $128 \mathrm{~d}$ feature vector;

d) Feature matching. After SIFT feature vectors are generated, use the nearest neighbors with Euclidean distance of the key feature vectors as a similarity judgment measures.

B. Speeded-up Robust Feature

SURF feature is an improvement to SIFT presented by Herbert Bay in 2006 [10]. It realized a speed gain by using a Hessian matrix-based measure approximately. Basic process are also four steps:

a) Establish a scale space. SURF adopts different box filter instead of Gaussian filter to obtain scale pyramid. The image size is constant, and change is the size of the filter;

b) Extract the feature points. Hessian matrix is used to determine the candidate points. Centered round feature points, accumulate the Haar wavelet response in a sliding fan window with radius of 6 (value for scale) and the opening Angle of $60^{\circ}$. The main direction is the direction of the greatest Haar accumulative value;

c) Generate the descriptor. Use feature points as the center, image size of the $20 \sigma \times 20 \sigma$ is divided into $4 \times 4$ blocks along the main direction. Each block calculates Haar wavelet response with template size of $2 \sigma$. Count the response value, and form a $64 \mathrm{~d}$ characteristic vector;

d) Match feature point. SURF is also matching based on threshold and the nearest neighbors.

SIFT feature points is more than the SURF, and the DOG can reflect closer to the image than the box function. SURF improve the speed of the detector by choosing the points with strong robustness. It achieved real-time computation with a little loss in performance, which represents an important advantage for many on-line computer vision applications.

\section{Simulation results}

The algorithms were tested with simulated data of 50 images. The sensitivity of the different matching features performance was examined. Simulated data were generated of remote sensing images with size of $300 \times 300$, noise variance of 0.8 , scale of $0 \sim 0.8$ and rotation angle of $0^{\circ} \sim 25^{\circ}$.

Camera parameters matrix is $Q=\left[\begin{array}{ccc}2831 & 0 & 2000 \\ 0 & 2831 & 1500 \\ 0 & 0 & 1\end{array}\right]$.

Observation angle for the satellite is between the direction vector to landmark and vector to star. The direction vector of the stars were measured by star sensor, its influence for error is not in consideration in this article. It is supposed to $\operatorname{be}(1 / \sqrt{2}, 0,1 / \sqrt{2})$ in experiment. Observed angle is obtained by the method presented in sec.2. Matching images by SURF and SIFT respectively in the condition that the noise variance of 0.2 , scale factor of 0.8 and rotation angle of $10^{\circ}$, the performance is shown in Table 1. And the result show that the method in sec. 2 can achieve a higher precision than traditional celestial navigation based on infrared sensor. 
Table.1. Angle Error of the method

\begin{tabular}{|c|c|c|c|c|}
\hline Features & Size & Rotation & Scale Factor & Angle Error \\
\hline SURT & $300 \times 300$ & $10^{\circ}$ & 0.8 & $6.0372^{\prime \prime}$ \\
\hline SIFT & $300 \times 300$ & $10^{\circ}$ & 0.8 & $5.8212^{\prime \prime}$ \\
\hline
\end{tabular}

Angular error results in condition of the noise variance of 0.2 , rotation angle of $10^{\circ}$ and different scale factors are shown in table 2:

Table.2. Results of Scale Test

\begin{tabular}{|c|l|l|}
\hline \multirow{2}{*}{ Scale Factor } & \multicolumn{2}{|c|}{ Angle Error } \\
\cline { 2 - 3 } & SURF & SIFT \\
\hline 0.5 & $42.69^{\prime \prime}$ & $11.11^{\prime \prime}$ \\
\hline 0.6 & $9.89^{\prime \prime}$ & $8.81^{\prime \prime}$ \\
\hline 0.7 & $7.52^{\prime \prime}$ & $7.75^{\prime \prime}$ \\
\hline 0.8 & $6.03^{\prime \prime}$ & $5.82^{\prime \prime}$ \\
\hline 0.9 & $4.93^{\prime \prime}$ & $4.83^{\prime \prime}$ \\
\hline
\end{tabular}

Angular error results of method in condition of the noise variance of 0.2 , scale factor of 0.8 and different rotation angle are shown in table 3:

Table.3. Results of Rotation Test

\begin{tabular}{|c|c|c|}
\hline \multirow{2}{*}{ Rotation Angle $^{\circ}{ }^{\circ}$ ) } & \multicolumn{2}{|c|}{ Angle Error } \\
\cline { 2 - 3 } & SURF & SIFT \\
\hline 5 & $5.31^{\prime \prime}$ & $4.92^{\prime \prime}$ \\
\hline 10 & $6.03^{\prime \prime}$ & $5.82^{\prime \prime}$ \\
\hline 15 & $8.98^{\prime \prime}$ & $7.86^{\prime \prime}$ \\
\hline 20 & $10.04^{\prime \prime}$ & $9.12^{\prime \prime}$ \\
\hline
\end{tabular}

In Table.2 and Table.3, we make comparisons between SURF and SITF. It is easy to find SIFT has a better performance on scale and rotation invariant than SURF.

In the test, matching method on SURF is five times as fast as SIFT. Even though SURF has a remarkable speed, but the speed in satellite navigation is not as important as in missile guidance. SIFT perform better in resistance of rotation and noise. So choose the SIFT features for our method at last.

\section{Conclusion}

The method presented in this work is autonomous navigation based on vision and star sensor. The method was tested with simulated and real data. Results show that the method based on image matching can achieve an accurate direction of landmark. And choose SIFT feature may loss efficiency but own a higher precision instead. 


\section{References}

[1] LIU Lin, WANG Haihong, HU Songjie. Summary on satellite orbit determination [J]. Journal of Spacecraft TT\&C Technology, 2005, 24(2):28-34.

[2] Chory M A, Hoffman D P, LeMay J L. Satellite autonomous navigation-status and history [J]. IEEE Position Location and Navigation Symposium, LasVegas, NV, Nov.4-7, 1986, 110-121.

[3] Fang JC, Ning XL. Principle and method of the spacecraft autonomous celestial navigation [M]. Beijing: National Defense Industry Press.2006.55-136.

[4] Betke. M, Gurvits. L. Mobile robot localization using landmarks. IEEE Trans. Robot. Autom. 13, 251-263 (1997).

[5] E. R. Hiller. Synthetic aperture radar map matching for navigation. Raytheon Company, Missile Systems Division, Bedford, MA01780, 1979, 92-100.

[6] L. Merino, F. Caballero, P. Forssen, et al. Multi-UAV Localization Based on Blob Features for Exploration Missions. IEEE Robotics and Automation Magazine, Vol.13, No. 3, September 2006.

[7] Bevilacqua A, Carozza L, and Gherardi A. An image registration approach for accurate satellite attitude estimation [C].Proceedings of the 5th International Symposium on Advances in Visual Computing: Part II, 2009: 827-836.

[8] LI Xiang, ZHU Zunshang, BU Yanlong, SHANG Yang. Vision-based satellite position measurement [J]. Journal of national university of defense technology, CS, 2014, 36(3), 63-67.

[9] David G. Lowe. Object recognition from local scale-invariant features [C]. The Proceedings of IEEE International Conference on Computer Vision. Corfu, Greece.1999:1150-1157.

[10] Bay H, Ess A, Tuytelaars T, et al. Speeded-up robust features (SURF) [J]. Computer Vision and Image Understanding,2008,7(3):346-359. 